\title{
SUR QUELQUES DIFFICULTÉS DE MESURES DES PERTES DE CHARGE DANS LES CONDUITES
}

\author{
par A. FORTIER \\ Protesseur à la Sorbonne \\ Ancien sous-directeur de l'Institut Polytechnique de Grenoble
}

Je voudrais rappeler rapidement la définition de la perte de charge dans une conduite pour: montrer ensuite que la grandeur ainsi définie est souvent difficilement mesurable en pratique. Les difficultés de mesure proviennent surtout de la non permanence des écoulements dans les conduites industrielles; pour effectuer des mesures précises on est donc conduit à enregistrer des pressions variables, je signalerai, pour terminer, un manomètre enregistreur simple qui paraît bien adapté à ce genre de mesures.

On avait coutume autrefois, dans les cours élémentaires d'électricité, d'employer des analogies hydrauliques pour expliquer les phénomènes électriques. Notre connaissance des lois du courant électrique étant actuellement beaucoup plus avancée que notre connaissance des lois des écoulements fluides, il est préférable aujourd'hui de faire l'inverse. Considérons par exemple deux grands réservoirs entre lesquels existe une différence de niveau constante $\mathrm{H}$ et reliés par une conduite alimentant une turbine. Soit $P$ le débit en poids dans la conduite, que nous supposerons constant, l'énergie mécanique perdue par l'eau par seconde dans la conduite et la turbine est égale au produit HP. L'installation électrique analogue comprend deux conducteurs entre lesquels existe une différence de potentiel $V$ et reliés par un fil conducleur alimentant un moteur, si I désigne l'intensité de courant, la puissance électrique dissipée dans le fil conducteur et le moteur est égale à VI. Soit maintenant deux points quelconques $A$ et $B$ du fil conducteur, si $V_{A}-V_{B}$ désigne la différence de potentiel entre les deux points $A$ et $B$ la puissance électrique perdue entre les points $A$ et $B$ est égale à $\left(V_{A}-V_{B}\right)$.

Par analogie, nous pouvons toujours mettre la puissance mécanique perdue par l'eau entre deux sections quelconques $A$ et $B$ de la conduite sous la forme du produit du débit en poids $P$ dans la conduite par une quantité homogène à une longueur et que nous désignerons par $\mathrm{H}_{\mathrm{A}}-\mathrm{H}_{\mathrm{B}}$

C'est la grandeur ainsi définie que nous appellerons perte de charge entre les sections $\mathrm{A}$ et B, cette grandeur possède les propriétés d'additivité des différences de potentiel et nous pouvons dire que la perte de charge entre les sections $A$ et $B$ est égale à la différence des charges $H_{A}$ et $\mathrm{H}_{\mathrm{B}}$ dans les deux sections $A$ et $B$

La quantité intéressante à connaître en pratique est la perte de puissance de l'eau dans la conduite, l'introduction de la perte de charge telle que nous venons de la définir n'a done un intérèt pratique qu'à condition de pouvoir exprimer cette perte de charge en fonction de quantités direetement mesurables : pressions, cotes et vitesses aux divers points des sections A et $B$.

Pour trouver cette expression de la perte de charge en fonction de grandeurs mesurables, on suppose, dans les cours élémentaires d'hydraulique, que le mouvement de l'eau dans la conduite est permanent et que la vitesse est constante en grandeur et direction dans une mème section. Une application simple du théorème des forces vives montre alors que la puissance mécanique W 
perdue par l'eau entre deux sections $A$ et $B$ peut effectivement se mettre sous la forme $\mathrm{IV}=\mathrm{P}\left(\mathrm{H}_{\mathrm{A}}-\mathrm{H}_{\mathrm{B}}\right)$ la charge $\mathrm{H}$ dans une section quelconque ayant l'expression classique :

$$
H=\frac{P}{\tilde{\omega}}+\Xi+\frac{\mathrm{V}^{2}}{2 g}
$$

p) désignant la pression au point de cote $\Xi$ de la section considérée, (i) le poidis spécifique de l'eau, V la vitesse de l'eau dans la section et $g$ l'accélération de la pesanteur.

On démontre par ailleurs que la quantité $\frac{\rho}{\sigma}+\Xi$ est constante dans toute la section et que cette quantité est mesurée par la cole du niveau de l'eau dans un tube piezométrique relié à une prise de pression statique située dans la section considérée.

Dans cette hypothèse simple, la mesure de la perte de charge entre deux sections se ramène donc à la mesure d'une différence de niveau entre deux tubes piezométriques, les vitesses dans les deux sections étant par ailleurs connues si on connaît le débit et les aires des deux sections. Si la conduite est de section constante ou si les vitesses sont suffisamment faibles pour qu'on puisse négliger le terme $\frac{\mathrm{V}}{2 g}$ la mesure de la perte de charge ne comporte qu'une mesure d'une différence de pression qu'on peut effectuer à l'aide d'un manomètre différentiel exactement comme on mesure une différence de potentiel à l'aide d'un voltmètre.

Nalheureusement, l'hypothèse d'un mouvement bien permanent et d'une répartition uniforme des vitesses dans les diverses sections de la conduite est loin d'être conforme à la réalité. La vitesse est toujours nulle sur la paroi et maximum au centre de la conduite. Si on suppose que les vitesses restent parallèles à une direction fixe, mais varient en grandeur d'un point à un autre d'une mème section, on démontre facilement que la charge $H$ conserve, pour un mouvement permanent, une expression identique à l'expression (1), le terme $\frac{\mathrm{V}^{2}}{2 g}$ est simplement multiplié par un coefficient $\alpha$ qui dépend de la répartition des vitesses ( $V$ désignant dans ce cas la vitesse moyenne dans la section quotient du débit en volume par l'aire de la section). Lorsque le coefficient $\alpha$ est peu différent de 1 , ce qui se produit en particulier pour les conduites cylindriques longues, on peut prendre en première approximation, $\alpha=1$, et on est ramené au cas de la répartition uniforme des vitesses. Les difficultés n’apparaissent donc que lorsqu'on veut tenir compte de la non permanence de l'écoulement.

Dans une conduite industrielle, les vitesses varient dans le temps d'une facon incessante en grandeul et direction, les pressions varient elles aussi et tous ceux qui ont fait des mesures de pression savent que les manomètres reliés à des prises de pression sur des conduites industrielles oscillent sans arrêt. La non permanence de l'écoulement est dûe soit à la turbulence, soit à des variations de régime des machines, soit plus généralement à l'instabilité de l'écoulement dans des singularités de l'installation : coudes, changements brusques de section, etc... Les lluctuations de pression dûes à la turbulence sont en général trop rapides pour être enregistrées par les manomètres usuels, tandis que les fluctuations de débit et de pression produites par l’instabilité de l'écoulement dans les singularités sont plus lentes et ce sont celles qu'on observe généralement.

La non permanence des écoulements industriels nous amène à reprendre la définition de la perte de charge. Remarquons tout d'abord que ce sont uniquement des grandeurs moyennes qu'il est intéressant de déterminer en pratique. Ce qu'il importe en effet de connaître, c'est l'énergie mécanique perdue par l'eau dans un tronçon de conduite pendant un temps suffisamment long. Soit W la puissance instantanée perdue par l'eau, pendant le temps $T$ l'énergie mécanique perdue sera $\int_{0}^{1} W d t$, ramenée à l'unité de temps, cette énergie correspond à une puissance moyenne quo 
nous noterons $\overline{\mathrm{W}}=\frac{1}{\mathrm{~T}} \int_{0}^{\mathrm{T}} \mathrm{W} d t$. Dans les cas usuels, cette puissance moyenne $\mathrm{W}$ ne dépend pas du temps, pourvu que le temps $\mathrm{T}$ soit long durant la période des fluctuations, nous dirons alors que l'écoulement est permanent en moyenne et nous raisonnerons uniquement dans ce qui suit, sur un cas particulier. Le débit de l'installation subit lui aussi des fluctuations dans le temps et nous définirons un débit moyen $\overline{\mathrm{P}}$ comme nous avons défini la puissance moyenne $\overline{\mathrm{W}}$. Nous appellerons alors perte de charge moyenne, entre deux sections $A$ et. $B$, une quantité que nous noterons $\mathrm{H}_{\mathrm{A}}-\mathrm{H}_{\mathrm{B}}$, et telle que :

$$
\overline{\mathrm{W}}=\left(\overline{\mathrm{H}}_{\mathrm{A}}-\overline{\mathrm{H}}_{\mathrm{B}}\right) \overline{\mathrm{P}}
$$

$\bar{H}_{A}$ et $\bar{H}_{B}$ désignent respectivement les charges moyennes dans les sections $A$ et $B$.

Ces définitions étant posées, il s'agit d'exprimer la charge moyenne dans une section en fonction de grandeurs moyennes mesurables, à savoir : pressions moyennes et vitesses moyennes aux divers points de la section. Or, dans le cas d'un écoulement extrêmement perturbé, il n'est pas possible de trouver de relation simple entre la charge moyenne et les grandeurs moyennes que nous venons de citer. L'expression générale de la charge moyenne est en effet de la forme :

$$
\overline{\mathrm{H}}=\frac{\iint_{s} \overline{\left(\frac{p}{\sigma}+\Xi+\frac{\mathrm{V}^{2}}{2 g}\right)} u d \sigma}{\overline{\mathrm{Q}}}
$$

$p$ et $\mathrm{V}$ désignant la pression et la vitesse instantanées au point de cote $\Xi$ centre de l'élément de surface $d_{\sigma}, \quad u$ la composante de la vitesse instantanée suivant la normale à $d_{\sigma}$ et $\overline{\mathrm{Q}}$ le débit moyen en volume. L'intégrale double est étendue à toute l'aire $S$ de la section, et la barre surmontant le produit $\left(\frac{P}{\tilde{\omega}}+\Xi+\frac{V}{2 g}\right) u$ indique qu'il faut prendre en chaque point la valeur. moyenne dans le temps de ce produit.

Pour calculer $\mathrm{H}$, il faut donc en toute rigueur enregistrer les variations de la pression et de la vitesse en un grand nombre de points de la section $S$ pour pouvoir calculer en chaque point la valeur moyenne du produit $\left(\frac{p}{\sigma}+\Xi+\frac{\mathrm{V}}{2 g}\right) u$. et on ne peut pas se contenter de mesurer séparément des pressions moyennes et des vitesses moyennes.

Dans le cas particulier où la répartition des pressions dans la section considérée est hydrostatique en moyenne, c'est-à-dire dans le cas où la valeur moyenne de $\frac{P}{\sigma}+\Xi$ ne dépend pas du point choisi dans la section, l'expression de la charge moyenne peut se mettre sous la forme :

$$
\overline{\mathbf{H}}=\frac{\bar{p}}{\bar{\omega}}+\Xi+\alpha \frac{\mathrm{U}^{2}}{2 g}+\bar{h}
$$

U désignant le quotient du débit moyen en volume par l'aire de la section, $\alpha$ un coefficient de répartition de vitesse peu différent de 1 si la répartition des vitesses moyennes dans la section s'éloigne peu de la répartition uniforme, et $h$ une quantité que nous n'expliciterons pas, mais qui tend vers zéro, lorsque l'amplitude des fluctuations de pression et de vitesse tend ellemême vers zéro.

Nous retrouverons dans ce cas une expression analogue à l'expression simple de la charge dans une section, lorsque l'écoulement est permanent, et nous voyons que pour qu'il soit possible de calculer la charge moyenne à partir des pressions et des vitesses moyennes, il faut que la répartition des pressions moyennes soit hydrostatique et que l'amplitude des fluctuations de pression et de vitesse soit suffisamment faible pour qu'on puisse négliger le terme $\bar{h}$. 
L'aperçu qui précède montre l'intérêt qu'il y a à effectuer des enregistrements de pression et de vitesse pour évaluer le terme $\vec{h}$ et par suite l'erreur que ron commet lorsqu'on calcule des pertes de charge uniquement à partir des pressions et des vitesses moyennes. C'est dans ce but que nous avons mis au point un manomètre enregistreur basé sur le principe classique du mano-

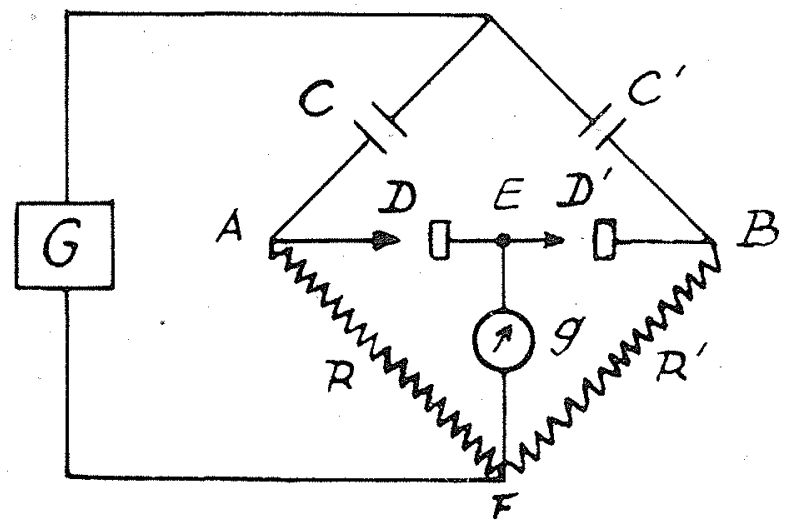
mètre à condensateur, mais qui comporte un montage élecirique simple que je voudrais signaler.

La capsule manométrique est constituće par une membrane métallique tendue formant l'armature mobile d'un condensateur C. Ce condensateur variable $C$ est intercalé dans un pont comprenant un condensateur fixe $\mathrm{C}^{\prime}$ el deux résistances $R$ et $R$ '. Dans la diagonale Al: du pont se trouvent deux diodes $\mathrm{D}$ et D'. Un galvanomètre enregistreur $g$ est intercalé entre les points $\mathrm{E}$ et $\mathrm{F}$, et le pont est alimenté par un générateur haute fréquence $G$.

Lorsque les capacités $\mathrm{C}$ et $\mathrm{C}^{\prime}$ 'sont égales, le galvanomètre $g$ reste au zéro, lorsque lat capacité C varie, le galvanomètre esí parcouru par un courant dont l'intensité est égale à la différence des intensités des courants redressés par les diodes $D$ et $D$, et le galvanomètre suit les variations de la capacité $\mathrm{C}$ et par suite les variations de la pression qui déforme la membrane constituant l'armature mobile du condensateur $C$.

Ce montage, très simple, est parfaitement stable, et il est susceptible de beaucoup d'autres applications, puisqu'il permet en fait d'enregistrer les variations de la distance entre les deux armatures du condensateur C. Comme manomètre enregistreur, il est d'un emploi beaucoup plus commode qu'un manomètre à quartz, ce dernier ne s̈imposant vraiment que pour l'enregistrement de iréquences très élevées.

D'une réalisation simple, ce manomètre peut être exécuté en un grand nombre d'exemplaires et permettre une étude systématique des pertes de charge en tenant compte des difficultés que nous avons signalées.

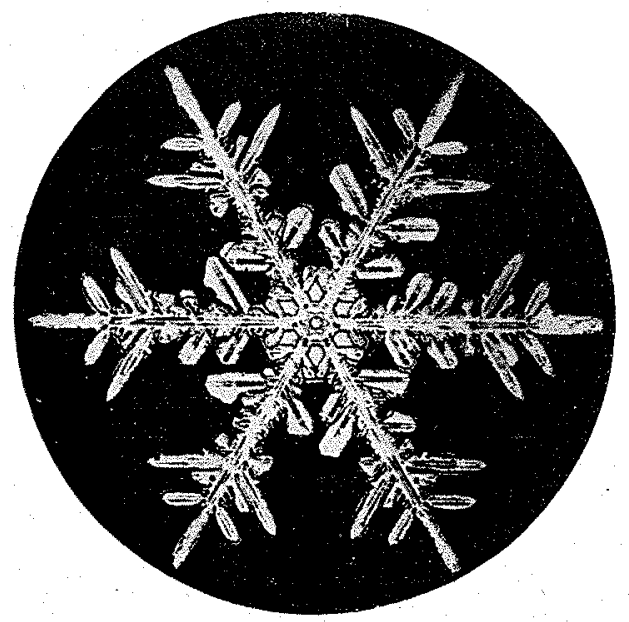

